\title{
Occupational tuberculosis exposures and conversion rates can guide deimplementation of annual tuberculosis skin test screening
}

\author{
Takaaki Kobayashi MD ${ }^{1}$, Holly Meacham $\mathrm{MSN}^{1}$, Mohammed Alsuhaibani MD ${ }^{1,2}$, Stephanie Holley MBA ${ }^{1}$, \\ Alexandre R. Marra MD ${ }^{1,3}$, Michael B. Edmond MDㄹ, Daniel J. Diekema MD', Patrick G. Hartley MB, BCh $^{1}$ and \\ Jorge L. Salinas MD ${ }^{1}$ \\ ${ }^{1}$ Department of Internal Medicine, University of lowa Hospital \& Clinics, lowa City, lowa, United States, ${ }^{2}$ Department of Pediatrics, College of Medicine, Qassim \\ University, Qassim, Saudi Arabia and ${ }^{3}$ Division of Medical Practice, Hospital Israelita Albert Einstein, São Paulo, Brazil
}

To the Editor-Tuberculosis (TB) is a serious health problem worldwide. However, TB incidence in the United States has declined, including among healthcare personnel (HCP). ${ }^{1}$ The Centers for Disease Control and Prevention (CDC) recently updated their recommendations for TB transmission prevention in healthcare facilities, suggesting discontinuing annual tuberculin skin tests (TSTs). ${ }^{2,3}$ However, healthcare facilities may continue annual TSTs based on local epidemiology. We assessed TB infection prevention and control parameters such as TB incidence, exposures, and transmission rates to guide the deimplementation of annual TSTs in our facility.

\section{Methods}

The University of Iowa Hospitals \& Clinics (UIHC) is an 811-bed tertiary-care hospital in a state with low $\mathrm{TB}$ incidence. ${ }^{4} \mathrm{We}$ calculated the frequency of TB cases, exposures, and TST conversions among HCPs. We retrieved data for patients with an International Classification of Disease, Tenth Edition (ICD-10) code for TB (A15 respiratory TB, A17 TB of the nervous system, A18 TB of other organs, and A19 miliary TB) from January 2016 to October 2019. We supplemented our search with microbiology data: positive culture or nucleic acid amplification test for Mycobacterium tuberculosis. We categorized TB cases via manual chart review: active, latent, previously treated, unclear history, or no TB. We defined potentially infectious $\mathrm{TB}$ as pulmonary cases and extrapulmonary cases that underwent an aerosol-generating procedure. Data on exposure events, exposed HCPs, and TST results were obtained from the employee health clinic. Conversion was defined as an increase in TST response by $\geq 10 \mathrm{~mm}$ within a 2 year period.

\section{Results}

During 2016-2019, we identified 207 suspected TB cases: 197 patients based on ICD-10 codes and an additional 10 patients via microbiology data; 48 had active TB (Fig. 1). Upon investigation, $31(65 \%)$ were deemed potentially infectious: 24 had pulmonary TB and 7 had extrapulmonary TB and underwent an aerosol-generating procedure at an infected body site. Among those, 10 cases (32\%) led to the exposures of $200 \mathrm{HCP}$. Baseline and postexposure TSTs were available for 179 of these HCP \footnotetext{
edu

Author for correspondence: Takaaki Kobayashi, E-mail: Takaaki-kobayashi@uiowa.

Cite this article: Kobayashi T, et al. (2021). Occupational tuberculosis exposures and conversion rates can guide deimplementation of annual tuberculosis skin test screening. Infection Control \& Hospital Epidemiology, 42: 123-124, https://doi.org/10.1017/ ice.2020.205
}

(C) 2020 by The Society for Healthcare Epidemiology of America. All rights reserved.
207 patients identifed by ICD codes and microbiology data
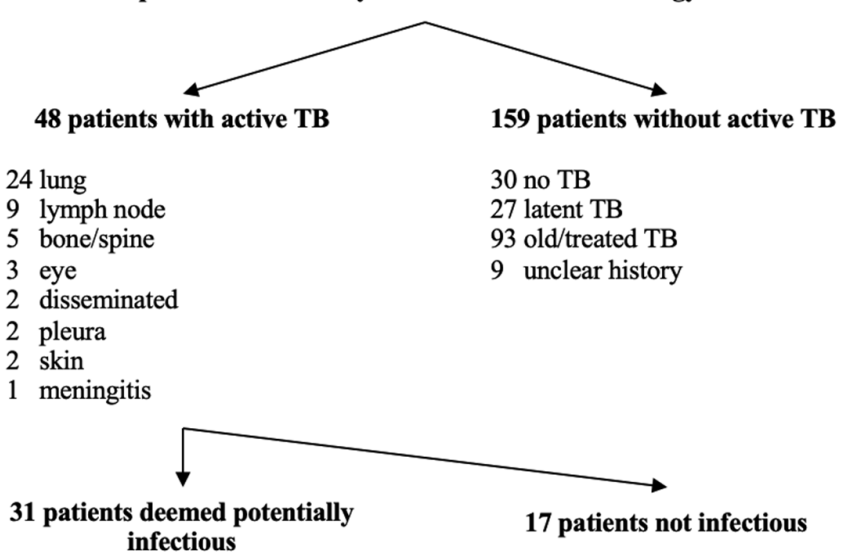

24 Pulmonary

7 Extra-pulmonary with an

aerosol generating procedure

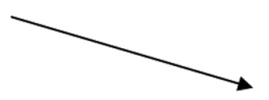

10 cases led to total 200 exposures

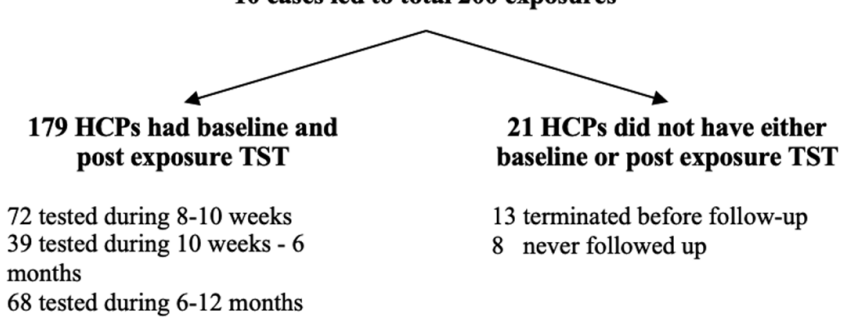

68 tested during 6-12 months

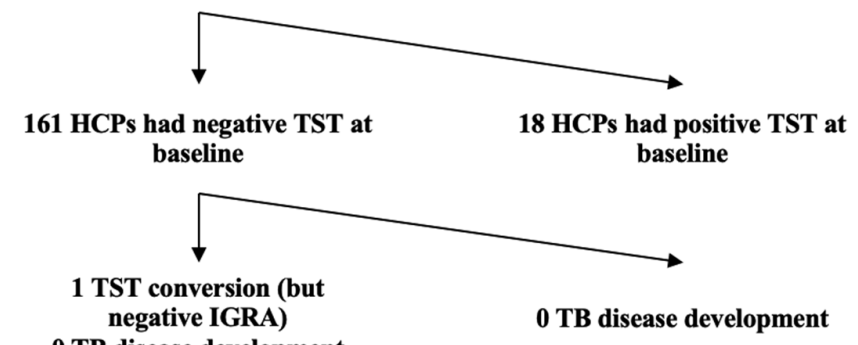

0 TB disease development

Fig. 1. Patient identification. Note. ICD, International Classification of Disease; TB, tuberculosis; HCP, healthcare personnel; TST, tuberculosis skin test; IGRA, interferon-gamma release assay. 
(88\%). Among them, 161 HCP (90\%) had a negative TST at baseline, and conversion was seen in only 1 case $(0.6 \%)$. This HCP was a nursing assistant exposed to a patient with pulmonary TB. However, a concurrent interferon-gamma release assay (IGRA) was negative, and no treatment was offered. Among $18 \mathrm{HCP}$ (10\%) with a positive TST at baseline, no HCP developed symptoms during the observation period. Regarding timing of postexposure TSTs, $111 \mathrm{HCP}(69 \%)$ had been followed at 6 months after the exposure.

\section{Discussion}

We used ICD-10 codes, microbiology, and employee health clinic data to examine TB infection control parameters in our institution. Nearly one-third of infectious TB cases led to the exposure of HCPs. However, the TST conversion rate was very low. Twothirds of HCPs had a post-exposure TST evaluation within 6 months after exposure. Exposure and conversion rates are useful indicators of TB infection control and may guide deimplementation of annual TSTs.

Our finding of one-third of TB cases potentially leading to HCP exposures is lower than previously reported. Kelly et $\mathrm{al}^{5}$ reported their TB contact investigation experience in a busy New York City Hospital and found that $58 \%$ of pulmonary TB cases led to exposures. Contrary to common belief, suspecting and promptly isolating TB cases may be easier in a low-incidence setting because sociodemographic factors classically associated with TB may be more apparent (eg, place of birth).

Our analysis confirmed a low TST conversion rate $(0.6 \%)$ at a referral and safety-net hospital in a low-incidence state. A recent systematic review indicated that conversions during serial testing ranged from $0.7 \%$ to $4 \%$ depending on whether TST or IGRA was used. $^{2}$ Deimplementing annual TSTs in facilities located in jurisdictions with a TB incidence higher than the national TB incidence (eg, California, Florida, Texas, or New York City) could be considered after confirming that institutional conversion rates are below the reported $\sim 0.7 \%-4 \%$ for the United States.

Two-thirds of HCPs (69\%) had a postexposure TST evaluation within 6 months after exposure. The remaining $31 \%$ had a subsequent TST during the annual screening process. Timeliness of TST evaluations after TB exposure is not commonly reported in the literature. Because the risk of developing TB disease is higher in the first 1-2 years following an exposure, ${ }^{6,7}$ prompt TST evaluations are important. The CDC recommends a TST 8-10 weeks after the last exposure. ${ }^{2}$ Hospitals deimplementing annual TB screening will need to enforce the timeliness of postexposure TST evaluations.

Our study has several limitations. It was a single-center study in a low-incidence setting; exposure and conversion rates may vary in facilities located in higher incidence states. Because our conversion rate was very low, we could not stratify by location (eg, emergency department, TB clinic). Hospitals with higher TB conversion rates may stratify and de-implement annual TSTs in clinical areas with lower conversion rates (eg, administrative personnel, outpatient clinics) and continue in clinical areas with conversion rates higher than expected.

In conclusion, exposure and conversion rates in healthcare facilities are useful indicators of TB infection control. They guided the deimplementation of annual TST in our facility according to the new CDC recommendations. A sustained effort to ensure timely exposure identification and contact investigations will be key to avoiding TB transmission in healthcare facilities.

\section{References}

1. Talwar A, Tsang CA, Price SF, et al. Tuberculosis—United States, 2018. Am J Transplant 2019;19:1582-1588.

2. Sosa LE, Njie GJ, Lobato MN, et al. Tuberculosis screening, testing, and treatment of US health care personnel: recommendations from the National Tuberculosis Controllers Association and CDC, 2019. Morbid Mortal Wkly Rep 2019;68:439-443.

3. Welbel SF, French AL, Bush P, DeGuzman D, Weinstein RA. Protecting healthcare workers from tuberculosis: a 10-year experience. Am J Infect Control 2009;37:668-673.

4. Reported tuberculosis in the United States, 2018. Centers for Disease Contorl and Prevention website. https://www.cdc.gov/tb/statistics/reports/2018/ default.htm. Published 2018. Accessed May 12, 2020.

5. Kelly AM, D’Agostino JF, Andrada LV, Liu J, Larson E. Delayed tuberculosis diagnosis and costs of contact investigations for hospital exposure: New York City, 2010-2014. Am J Infect Control 2017;45:483-486.

6. Poulsen A. Some clinical features of tuberculosis. 1. Incubation period. Acta Tuberc Scand 1950;24:311-346.

7. Wallgren A. The time-table of tuberculosis. Tubercle 1948;29:245-251. 